hep-ph/0001069

TMUP-HEL-9907

\title{
Matter effects on neutrino oscillations in gravitational and magnetic fields
}

\author{
H. Athar \\ Department of Physics, Tokyo Metropolitan University, \\ Minami-Osawa 1-1, Hachioji, Tokyo 192-0397, \\ Japan \\ José F. Nieves \\ Laboratory of Theoretical Physics \\ Department of Physics, P.O. Box 23343 \\ University of Puerto Rico, Río Piedras \\ Puerto Rico 00931-3343
}

June 1999

\begin{abstract}
When neutrinos propagate in a background, their gravitational couplings are modified by their weak interactions with the particles in the background. In a medium that contains electrons but no muons or taons, the matter-induced gravitational couplings of neutrinos are different for the various neutrino flavors, and they must be taken into account in describing the phenomena associated with the neutrino oscillations in the presence of strong gravitational fields. Here we incorporate those couplings in that description, including also the effects of a magnetic field, and consider the implications that they have for the emission of high energy neutrinos in the vicinity of Active Galactic Nuclei.
\end{abstract}

\section{Introduction}

It is now well known that the interactions of a neutrino with the background particles can influence the neutrino properties in essential ways as it propagates through a medium. Those effects can have important consequences for various physical phenomena, such as the Mikheyev-Smirnov-Wolfenstein (MSW) mechanism and its variations in the context of the solar neutrino problem, the explanation of the large velocities of pulsars in terms of the asymmetric emission of neutrinos from the proto neutron star and the propagation of high energy $\left(E \geq 10^{6} \mathrm{GeV}\right)$ neutrinos in the vicinity of cores of Active Galactic Nuclei (AGN) [1].

In all these applications, a common ingredient is the fact that the various neutrino flavors interact with the background particles differently. While the standard neutrino interactions in the fundamental Lagrangian have a universal character, the universality is broken when the effects of the medium are taken into account.

In the presence of a medium, these breakdown of the universality of the neutrino interactions includes also the gravitational ones. In Ref. [2] the effective gravitational vertex of neutrinos was determined by calculating the one-loop contribution to the neutrino stress-energy tensor. In the presence of a static gravitational potential, such matter-induced interactions lead to an additional contribution to the neutrino dispersion relations, or equivalently their indices of refraction, which depend on the gravitational potential and are not the same for all the neutrino flavors. As was stressed in that work, those calculations were based on the standard model of particle interactions and the commonly accepted linearized theory of gravity with a universal tree-level coupling. The non-universal character of the induced gravitational couplings is a consequence of the flavor asymmetry of the background and not of an assumed breakdown of universality of the fundamental Lagrangian.

On the other hand, it has been observed by Píriz, Roy and Wudka [3], that high energy neutrinos originating from AGN can have resonant spin-flavor transitions due to the combined effects of the gravitational interactions 
and the presence of a large magnetic field. AGN can be a source of high energy neutrinos and, therefore, a good understanding of such transitions is useful to estimate the expected fluxes from these objects in the forthcoming searches for high energy neutrinos in neutrino telescopes.

In the calculations of Ref. [3], the matter, magnetic and gravitational effects were considered. However, the latter were introduced using the standard tree-level couplings of neutrinos to gravity and, in particular, the effects of the medium on the effective gravitational interactions were not taken into account. This amounts to neglect the additional matter-induced gravitational contribution to the neutrino dispersion relation. As we have already mentioned, they depend on the gravitational potential and, what is more important for the issue of resonant transitions, are not flavor symmetric. In contrast, the tree-level gravitational couplings are the same for all the neutrino flavors, including the right handed (singlet) neutrinos, and hence should have no effect whatsoever on the phenomenon of neutrino oscillations.

Motivated by these considerations, in this work we take another look at the subject of neutrino oscillations in the presence of a gravitational field. Our objective is to pay particular attention to the effects of the matter-induced gravitational couplings that we have already mentioned, with a view to their implications in the context of high energy neutrino emission from AGN.

We start in Section 2 with a brief overview of the main results obtained in Ref. [2] that are needed in subsequent sections here. This includes a summary of the formulas obtained there for the dispersion relations for standard left-handed neutrinos in the presence of a gravitational potential, taking into account the matter-induced terms in the gravitational vertex of the neutrino. In Section 3 we set up in general terms the equations that are relevant for treating the phenomenon of resonant neutrino (spin)-flavor transitions in the presence of a gravitational potential. The treatment includes the possibility that a magnetic field may also be present, under the assumption that the neutrinos have an intrinsic magnetic moment. Using the results of Section 3 as the starting point, in Section 14 we study the effect of the resonant transitions on the the determination of the flux of high energy neutrinos emitted by AGN, and finally the conclusions are given in Section 5 .

\section{Matter effects on the neutrino gravitational interactions}

In Ref. [2] we obtained the dispersion relation that is obeyed by a standard left-handed neutrino in the presence of a static gravitational potential. In this section we review briefly those results and consider the corresponding ones for a right-handed (singlet) neutrino .

As already indicated, those results were derived in the linearized theory of gravity, in which the metric tensor is written as

$$
g_{\lambda \rho}=\eta_{\lambda \rho}+2 \kappa h_{\lambda \rho}
$$

where $\eta_{\lambda \rho}$ is the flat space metric. We then expand the Lagrangian in the presence of gravity in powers of $\kappa$ and keep only the first order terms. In this formulation, $h_{\lambda \rho}$ appears as the graviton field, which is a spin- 2 quantum field coupled to the stress-energy tensor, whose interactions can be studied in the flat Minkowskian background. The Einstein-Hilbert action for pure gravity is given by

$$
\mathcal{A}=\frac{1}{16 \pi G} \int d^{4} x \sqrt{-\mathrm{g}} R
$$

where $R$ is the Ricci scalar, $\mathrm{g}$ is the determinant of the matrix $g_{\lambda \rho}$, and $G$ is the Newton's constant. Using Eq. (2.1), we can verify that this gives the correct kinetic terms for the spin-2 field if we make the identification

$$
\kappa=\sqrt{8 \pi G} .
$$

Then starting from the Dirac Lagrangian for a given fermion $f$ in the presence of gravity, the coupling of the graviton field $h_{\lambda \rho}$ with the fermion field can be written as

$$
\mathcal{L}_{h}^{(f f)}=-\kappa h^{\lambda \rho}(x) \widehat{T}_{\lambda \rho}^{(f)}(x),
$$

where the stress-energy tensor operator $\widehat{T}_{\lambda \rho}^{(f)}$ for the fermion field is given by

$$
\widehat{T}_{\lambda \rho}^{(f)}(x)=\left\{\frac{i}{4} \bar{\psi}(x)\left[\gamma_{\lambda} \partial_{\rho}+\gamma_{\rho} \partial_{\lambda}\right] \psi(x)+H . c .\right\}-\eta_{\lambda \rho} \mathcal{L}_{0}^{(f)}(x) .
$$


Here $\mathcal{L}_{0}^{(f)}(x)$ is the Lagrangian for the free Dirac field, which we write in the explicitly Hermitian form

$$
\mathcal{L}_{0}^{(f)}=\left[\frac{i}{2} \bar{\psi} \gamma^{\mu} \partial_{\mu} \psi+H . c .\right]-m_{f} \bar{\psi} \psi .
$$

From Eqs. 2.4) and (2.5) it follows that the term associated with the gravitational fermion vertex in a Feynman diagram is $-i \kappa V_{\lambda \rho}^{(f)}$, where

$$
V_{\lambda \rho}^{(f)}\left(p, p^{\prime}\right)=\frac{1}{4}\left[\gamma_{\lambda}\left(p+p^{\prime}\right)_{\rho}+\gamma_{\rho}\left(p+p^{\prime}\right)_{\lambda}\right]-\frac{1}{2} \eta_{\lambda \rho}\left[\left(\not p-m_{f}\right)+\left(\not p^{\prime}-m_{f}\right)\right] .
$$

It is easy to deduce by inspection that for a left $\left(\nu_{L}\right)$ or a right $\left(\nu_{R}\right)$ handed massless neutrino, the corresponding quantity is given by

$$
V_{\lambda \rho}^{\left(\nu_{L, R}\right)}\left(k, k^{\prime}\right)=\frac{1}{4}\left[\gamma_{\lambda}\left(k+k^{\prime}\right)_{\rho}+\gamma_{\rho}\left(k+k^{\prime}\right)_{\lambda}\right] \chi-\frac{1}{2} \eta_{\lambda \rho}\left[\not k+\not k^{\prime}\right] \chi,
$$

where $\chi=L, R \equiv \frac{1}{2}\left(1 \mp \gamma_{5}\right)$.

When the effects of the background medium are taken into account, the result is that the matrix element of the total stress-energy tensor $\widehat{T}_{\mu \nu}(x)$ between neutrino states, with incoming and outgoing momenta $k$ and $k^{\prime}$ respectively, is given by

$$
\left\langle\nu_{L, R}\left(k^{\prime}\right)\left|\widehat{T}_{\mu \nu}(0)\right| \nu_{L, R}(k)\right\rangle=\bar{u}_{L, R}\left(k^{\prime}\right)\left(V_{\mu \nu}^{\left(\nu_{L, R}\right)}\left(k, k^{\prime}\right)+\Lambda_{\mu \nu}^{\left(\nu_{L, R}\right)}\left(k, k^{\prime}\right)\right) u_{L, R}(k) .
$$

The quantity $\Lambda_{\mu \nu}^{\left(\nu_{L}, R\right)}\left(k, k^{\prime}\right)$ represents the matter-induced contribution and its calculation to one-loop was the subject of Ref. [2]. That calculation was performed for the left-handed massless neutrinos, adopting the standard electroweak couplings for the neutrinos and the other particles. The corresponding result for the right-handed (singlet) neutrinos, since they have no standard couplings to matter, is

$$
\Lambda_{\mu \nu}^{\left(\nu_{R}\right)}=0 .
$$

The particular formulas for $\Lambda_{\mu \nu}^{\left(\nu_{L}\right)}$ are not relevant for us here. More important is the result that they imply for the dispersion relation that a neutrino obeys in the presence of a static gravitational potential $\phi^{e x t}$. Using Eq. (2.9) as the starting point, it was shown in Ref. [2] that the dispersion relation of a standard left-handed neutrino that propagates with momentum $\vec{K}$ is given by

$$
\omega_{K}^{(L)}=K+2 K \phi^{e x t}+b_{\mathrm{mat}}+b_{G},
$$

while for its antiparticle (a right-handed antineutrino) the sign in front of $b_{\text {mat }}$ and $b_{G}$ is the opposite. The coefficient $b_{\text {mat }}$ is the usual Wolfenstein term while $b_{G}$ represents the matter-induced gravitational contribution. Following the same arguments, it follows that the dispersion relation $\omega_{K}^{(s)}$ for a sterile neutrino (either left- or right-handed) is simply

$$
\omega_{K}^{(s)}=K+2 K \phi^{e x t} .
$$

It is therefore clear that the tree-level gravitational neutrino couplings, which are represented in Eqs. (2.11) and (2.12) by the $2 K \phi^{e x t}$ term, do not play any role whatsoever in the phenomenon of neutrino oscillations, since they appear as a common factor in the dispersion relations of all the neutrino states. In contrast, the matter-induced terms, which are zero for the right-handed singlet neutrinos and do not have the same value for the three lefthanded neutrino flavors, affect the neutrino oscillations via the MSW mechanism[4]. In those situations in which the gravitational contribution can be neglected, Eq. 2.11) reduces simply to the Wolfenstein formula. However, in environments in which a relatively strong gravitational potential is present, the matter-induced gravitational term is important and must be taken into account in any discussion of MSW-type effects under such conditions. This is the subject we take up next. 


\section{Oscillations in a gravitational and a magnetic field}

We consider two neutrino families, the first of which we take to be $\nu_{e}$, and denote the second one by $\nu_{x}$, which can be either one of $\nu_{\mu, \tau}$ or a sterile neutrino $\nu_{s}$. Each family consists of a left-handed neutrino field $\nu_{a L}(a=e, x)$, and a corresponding right-handed partner $N_{a R}$ that we take to be a weak singlet. In addition to the mixing via the mass matrix, we allow the possibility that the neutrinos have an intrinsic magnetic moment coupling. In situations in which a magnetic field is present, those couplings can induce the (resonant) spin transitions between the two neutrino helicity states. We consider two situations separately, according to the type of the magnetic moment coupling that they may have.

Case I Here we assume that the neutrinos have a magnetic moment interaction defined by the term

$$
L^{\prime}=-\frac{1}{2} \sum_{a, b} \mu_{a b} \bar{N}_{a R} \sigma_{\mu \nu} \nu_{b L} F^{\mu \nu}+H . c .
$$

in the Lagrangian. As shown in the Appendix, if the neutrino flavor amplitudes are assembled in the vector

$$
\chi=\left(\begin{array}{c}
\alpha_{\nu_{e L}} \\
\alpha_{\nu_{x L}} \\
\beta_{\nu_{e R}} \\
\beta_{\nu_{x R}}
\end{array}\right)
$$

then their evolution is governed by the Hamiltonian matrix

$$
H=\left(\begin{array}{cc}
K+b_{\mathrm{mat}}+b_{G}+\frac{\left(m+\mu B_{\|}\right)^{2}}{2 K} & -\mu B_{T} \\
-\mu B_{T} & K+\frac{\left(m-\mu B_{\|}\right)^{2}}{2 K}
\end{array}\right),
$$

where each entry in this equation is itself a $2 \times 2$ matrix. In particular, $m$ is the neutrino mass mixing matrix and $\mu$ is the magnetic moment matrix defined in Eq. (3.1), while $K$ denotes the magnitude of the neutrino momentum and $B_{\|}, B_{T}$ are the components of the magnetic field parallel and transverse to $\hat{K}$, respectively. The terms $b_{\text {mat }}$ and $b_{G}$ are diagonal matrices whose values depend on the composition of the background medium. At this point it is useful to introduce the particle and antiparticle momentum distribution functions

$$
f_{f, \bar{f}}\left(p_{f}\right)=\frac{1}{e^{\beta\left(E_{f} \mp \mu_{f}\right)}+1},
$$

where the upper and the lower signs hold for the particle and the antiparticle respectively, $\mu_{f}$ is the chemical potential of the fermion $f$ and

$$
p_{f}^{\mu}=\left(E_{f}, \vec{P}\right), \quad E_{f}=\sqrt{\vec{P}^{2}+m_{f}^{2}} .
$$

The corresponding total number densities are given by

$$
n_{f}=2 \int \frac{d^{3} P}{(2 \pi)^{3}} f_{f}, \quad n_{\bar{f}}=2 \int \frac{d^{3} P}{(2 \pi)^{3}} f_{\bar{f}}
$$

In terms of these quantities,

$$
b_{\text {mat }}= \begin{cases}b_{e}+\sum_{f} X_{f} b_{f} & \text { for } \nu_{e} \\ \sum_{f} X_{f} b_{f} & \text { for } \nu_{\mu}, \nu_{\tau} \\ 0 & \text { for } \nu_{s}\end{cases}
$$


and

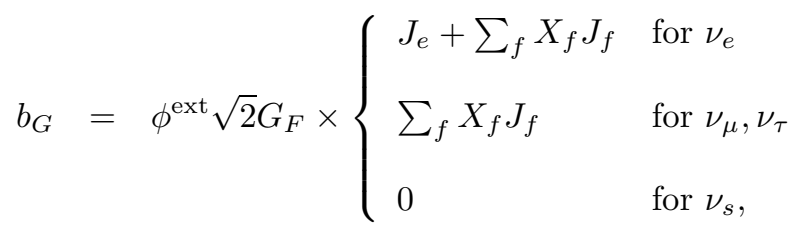

where

$$
\begin{aligned}
b_{f} & =\sqrt{2} G_{F}\left(n_{f}-n_{\bar{f}}\right), \\
J_{f} & =-3\left(n_{f}-n_{\bar{f}}\right)+\int \frac{d^{3} P}{(2 \pi)^{3} 2 E_{f}} \frac{d F_{f}}{d E_{f}},
\end{aligned}
$$

with

$$
F_{f}=4\left(2 E_{f}^{2}-m_{f}^{2}\right)\left(f_{f}-f_{\bar{f}}\right) .
$$

In Eqs. (3.7) and (3.8), the sum over $f$ must be made with respect to all the particle species that compose the background, and $X_{f}$ stands for their neutral current couplings. So, for example, for a background composed of electrons, neutrons, protons and their antiparticles $(f=e, n, p)$,

$$
\begin{aligned}
-X_{e}=X_{p} & =\frac{1}{2}-2 \sin ^{2} \theta_{W}, \\
X_{n} & =-\frac{1}{2} .
\end{aligned}
$$

The explicit formulas for $J_{f}$ were given in Ref. [2] for various limiting cases.

Case II In this case we assume that the magnetic moment interaction term is of the form[5]

$$
L^{\prime}=-\frac{1}{2} \mu \bar{\nu}_{x R}^{c} \sigma_{\mu \nu} \nu_{e L} F^{\mu \nu}+\text { H.c. },
$$

but that the mass terms in the Lagrangian are diagonal, i.e.,

$$
L_{m}=-m_{\nu_{e}} \bar{N}_{e R} \nu_{e L}-m_{\nu_{x}} \bar{N}_{x R} \nu_{x L}+H . c .,
$$

so that there is no mixing in the mass matrix. Denoting by $\alpha_{\nu_{e}}$ and $\beta_{\bar{\nu}_{x}}$ the $\nu_{e L}$ and $\nu_{x R}^{c}$ components of the wave function, and writing them in the form

$$
\chi=\left(\begin{array}{c}
\alpha_{\nu_{e L}} \\
\beta_{\bar{\nu}_{x R}}
\end{array}\right)
$$

the Hamiltonian matrix that determines their evolution is given in this case by 6

$$
H=\left(\begin{array}{cc}
K+b_{\mathrm{mat}}^{\left(\nu_{e}\right)}+b_{G}^{\left(\nu_{e}\right)}+\frac{m_{\nu_{e}}^{2}+\frac{\mu^{2} B_{\|}{ }^{2}}{2 K}}{2 K} & -\mu B_{T} \\
-\mu B_{T} & K-b_{\operatorname{mat}}^{\left(\nu_{x}\right)}-b_{G}^{\left(\nu_{x}\right)}+\frac{m_{\nu_{x}}^{2}}{2 K}+\frac{\mu^{2} B_{\|}{ }^{2}}{2 K}
\end{array}\right),
$$

where $b_{\text {mat }}^{\left(\nu_{a}\right)}$ and $b_{G}^{\left(\nu_{a}\right)}(a=e, x)$ are given in Eqs. (3.7) and (3.8).

Notice that, in the MSW mechanism involving only standard left-handed neutrinos, the matter effects due to the neutral current interactions are the same for all the neutrino species and therefore are not relevant as far as the oscillation mechanism is concerned. In contrast, in the situation we have just considered, those neutral current contributions have opposite sign for $\nu_{e L}$ and $\bar{\nu}_{\mu, \tau R}^{c}$, and are zero for $\nu_{s}$, and therefore must be taken into account. In the physical settings that we are considering, they can be important since they involve the nucleons and their couplings to gravity.

Eqs. (3.3) and (3.15) set the framework for our consideration of the possible effects that the matter-induced gravitational interactions of the neutrinos may have for the phenomenon of (resonant) transitions in the presence of a strong gravitational field. As a concrete example, we consider those transitions in the environment of AGN. 


\section{Resonant transitions in the vicinity of AGN}

For concreteness, we focus our attention on possible transitions between the $\nu_{e}$ and $\nu_{\tau}$ flavors for the following reason. Some galaxies have relatively bright centers (as compared to the total photon luminosity of the whole galaxy). High energy photons reaching tens of thousands of $\mathrm{GeV}$ have been observed from these centers. If Fermi mechanisms are responsible for accelerating the electrons in these systems, then protons are also expected to be accelerated by similar ones. If this is true, neutrinos are expected to be produced in $p \gamma$ or/and $p p$ collisions, providing the signature for proton acceleration. Tau neutrinos are also to be produced in the same collisions but at highly suppressed levels, because both the branching ratios and the production rates for unstable hadrons that decay into tau neutrinos are much lower than for muon or electron neutrinos. These two (suppression) factors enter into the calculation of relevant intrinsic neutrino fluxes multiplicatively, implying that the intrinsic $\nu_{\tau}$ (and $\bar{\nu}_{\tau}$ ) flux is relatively rather small. This $\nu_{\tau}$ (and $\bar{\nu}_{\tau}$ ) flux may possibly be somewhat enhanced due to (resonant) transitions between relevant neutrino states.

Briefly, in $p \gamma$ collisions, the protons and photons may give rise to high energy $\nu_{\tau}$ (and $\bar{\nu}_{\tau}$ ) mainly through $p+\gamma \rightarrow D_{S}^{+}+\Lambda^{0}+\bar{D}^{0}$ in addition to producing $\nu_{e}$ and $\nu_{\mu}$ mainly through $p+\gamma \rightarrow \Delta^{+} \rightarrow n+\pi^{+}$. The production cross-section for $D_{S}^{+}$is essentially up to three orders of magnitude lower than that of $\Delta^{+}$production for the relevant center of mass energy scale. Moreover the branching ratio of $D_{S}^{ \pm}$to decay eventually into $\nu_{\tau}\left(\bar{\nu}_{\tau}\right)$ is approximately two orders of magnitude lower than for $\Delta^{+}$to subsequently decay into $\nu_{e}$ and $\nu_{\mu}$ through $\pi^{+}$. These two suppression factors along with the relevant kinematic limits give approximately the ratio of intrinsic fluxes of tau neutrinos and electron neutrinos as $F^{0}\left(\nu_{\tau}+\bar{\nu}_{\tau}\right) / F^{0}\left(\nu_{e}+\bar{\nu}_{e}\right) \sim 10^{-5}$.

In $p p$ collisions, the $\nu_{\tau}$ flux may be obtained through $p+p \rightarrow D_{S}^{+}+X$. The relatively small cross-section for $D_{S}^{+}$ production together with the low branching ratio into $\nu_{\tau}$ implies that the $\nu_{\tau}$ flux in $p p$ collisions is also suppressed up to $4-5$ orders of magnitude relative to $\nu_{e}$ and/or $\nu_{\mu}$ fluxes.

Thus, in both type of collisions, the estimated intrinsic $\nu_{\tau}$ flux from the AGNs is rather small relative to $\nu_{e}$ and/or $\nu_{\mu}$ fluxes, essentially for the entire relevant neutrino energy range $\left(2 \cdot 10^{6} \leq \mathrm{K} / \mathrm{GeV} \leq 2 \cdot 10^{7}\right)$ [7]. However, as we point out later in this Section, due to transitions between relevant neutrino flavors, it is possible that $F^{0}\left(\nu_{\tau}+\right.$ $\left.\bar{\nu}_{\tau}\right) / F^{0}\left(\nu_{e}+\bar{\nu}_{e}\right) \gg 10^{-5}$, thus raising the possibility of detection of high energy $\nu_{\tau}$ in new $\mathrm{km}^{2}$ surface area neutrino telescopes within the relevant neutrino energy range [8].

We first briefly describe the matter density and magnetic field profiles in the AGN. According to [9], the matter density profile in the AGN is given as a function of the distance $r$ from the center by

$$
\rho_{A G N}(r)=\rho_{0} f(x)
$$

where $\rho_{0} \sim 1.4 \cdot 10^{-12} \mathrm{~g} \mathrm{~cm}^{-3}$ (we take the typical luminosity of the AGN to be $\sim 10^{45} \mathrm{erg} \mathrm{s}^{-1}$ ) and $f(x)=$ $x^{-2.5}\left(1-0.1 x^{0.31}\right)^{-1}$ for $x \sim(10-100)$. Here $x \equiv r / R_{S}$, where $R_{S}$ is the Schwarzchild radius $R_{S} \simeq 3 \cdot 10^{11}\left(\frac{M_{A G N}}{10^{8} M_{\odot}}\right) \mathrm{m}$, and in terms of it the gravitational potential is expressed as $\phi^{e x t}=-1 / 2 x$. We consider the following magnetic field profile in the AGN [9]

$$
B_{A G N}=B_{0} g(x),
$$

where $B_{0} \sim 1.7 \cdot 10^{5} \mathrm{G}$ and $g(x)=x^{-1.75}\left(1-0.1 x^{0.31}\right)^{-0.5}$. We will use these matter density and magnetic field profiles in our estimates as an example.

In what follows, the discussion of resonant transitions in the AGN is divided in two parts, according to whether or not the magnetic field terms are important. We discuss the two necessary conditions for resonant transitions to occur in these two situations, namely, the level crossing and the adiabaticity at the level crossing, and we also comment briefly on the possibility of transitions between active and sterile neutrinos.

\subsection{No magnetic field interactions}

In this situation, the mixing occurs via the mass matrix only. After subtracting a term proportional to the identity matrix in Eq. (3.3), the relevant $2 \times 2$ Hamiltonian matrix in the $\left(\nu_{e L}, \nu_{\tau L}\right)$ basis is given by

$$
H_{F}=\frac{1}{2}\left(\begin{array}{cc}
\Delta H_{F} & \left(\Delta m_{e \tau}^{2} / 2 K\right) \sin 2 \theta_{e \tau} \\
\left(\Delta m_{e \tau}^{2} / 2 K\right) \sin 2 \theta_{e \tau} & -\Delta H_{F}
\end{array}\right),
$$

with

$$
\Delta H_{F}=\Delta V_{F}-\frac{\Delta m_{e \tau}^{2}}{2 K} \cos 2 \theta_{e \tau}
$$


where $\theta_{e \tau}$ is the vacuum mixing angle, $\Delta m_{e \tau}^{2}=m_{\nu_{2}}^{2}-m_{\nu_{1}}^{2}$ is the mass squared difference between the two neutrino mass eigenstates $\nu_{i L}(i=1,2)$, and

$$
\Delta V_{F} \simeq \sqrt{2} G_{F}\left(n_{e}+J_{e} \phi^{e x t}\right) .
$$

In contrast to the term proportional to the density $n_{e}$ in Eq. (4.5), the term proportional to the gravitational potential depends on the nature of the electron gas. For the case of a classical non-relativistic $\left(\beta^{-1} \equiv T \ll m_{e}\right)$ electron background [2],

$$
J_{e}=-\beta m_{e} n_{e} .
$$

In the following estimates we use $T \simeq(1-10) \mathrm{eV}$ as an example, which is the value that corresponds to the black body (thermal) spectrum temperature for the photons mentioned above [9]. This value of $T$ may be considered as the average temperature for the remaining background particles as well because of thermal equilibrium (it is mainly this ultraviolet bump in photon spectrum that interacts with the Fermi accelerated protons producing the relevant unstable hadrons).

Using the matter density profile given by Eq. (4.1) and for $K \sim 7 \cdot 10^{6} \mathrm{GeV}$, the level crossing condition $\left(\Delta H_{F}=0\right)$ can be satisfied for $\Delta m_{e \tau}^{2} \sim\left(10^{-10}-10^{-11}\right) \mathrm{eV}^{2}$. Without taking into account the (matter induced) gravity effects, the level crossing condition can also be satisfied but for values of $\Delta m_{e \tau}^{2}$ that are approximately two orders of magnitude smaller than the one just quoted. However, the other essential condition, namely, the adiabaticity at the level crossing 10

$$
\kappa_{F} \equiv\left(\frac{\Delta m_{e \tau}^{2}}{2 K}\right)^{2} \frac{2}{\pi} \frac{\sin ^{2} 2 \theta_{e \tau}}{\left|\Delta \dot{V}_{F}\right|} \geq 1,
$$

(where $\Delta \dot{V}_{F} \equiv \mathrm{d}\left(\Delta V_{F}\right) / \mathrm{d} r$ ) is not satisfied; that is, $\kappa_{F} \ll 1$. Therefore, the matter induced gravity effects do not lead to resonant flavor transitions between $\nu_{e}$ and $\nu_{\tau}$ ?.

\subsection{With magnetic field interactions}

There are several possible cases that can be considered, depending on the mass mixing matrix and the magnetic moment couplings. We consider the specific situation in which the $\nu_{e L}$ and $\nu_{\tau R}^{c}$ fields are mixed by a transition magnetic moment interaction without mixing in the mass matrix, as described in Case II in Section 3. In this case, after subtracting the term proportional to the identity in Eq. (3.15) the relevant Hamiltonian matrix in the $\left(\nu_{e L}, \nu_{\tau R}^{c}\right)$ basis can be written as

$$
H_{S F}=\frac{1}{2}\left(\begin{array}{cc}
\Delta H_{S F} & -2 \mu B_{T} \\
-2 \mu B_{T} & -\Delta H_{S F}
\end{array}\right),
$$

where

$$
\Delta H_{S F}=\Delta V_{S F}-\frac{\Delta m_{e \tau}^{2}}{2 K}
$$

with

$$
\Delta V_{S F} \simeq \sqrt{2} G_{F}\left[\left(1+2 X_{e}\right)\left(n_{e}+J_{e} \phi^{e x t}\right)+2 \sum_{f=n, p} X_{f}\left(n_{f}+J_{f} \phi^{e x t}\right)\right]
$$

and $\Delta m_{e \tau}^{2}=m_{\nu_{\tau}}^{2}-m_{\nu e}^{2}$. For simplicity of the notation, in the reminder of this section we take a purely transverse magnetic field, so that $B_{T}=B$. In the more general case, the formulas given below hold with the replacement

\footnotetext{
${ }^{1}$ For the channel $\nu_{\mu} \rightarrow \nu_{\tau}$ the matter effects are absent, but there is an interesting possibility of vacuum flavor oscillations as supported by the recent superkamiokande data 11. The vacuum flavor oscillation probability expression is given by the familiar expression$$
P\left(\nu_{\mu} \rightarrow \nu_{\tau}\right)=\sin ^{2} 2 \theta_{\mu \tau} \sin ^{2}\left(\frac{\Delta m_{\mu \tau}^{2}}{4 K} L\right) .
$$

where $\theta_{\mu \tau}$ is the relevant vacuum mixing angle with the corresponding mass squared difference $\Delta m_{\mu \tau}^{2}$. We take $\Delta m_{\mu \tau}^{2}$ and $\sin ^{2} 2 \theta_{\mu \tau}$ values $\left(\Delta m_{\mu \tau}^{2} \sim 10^{-3} \mathrm{eV}^{2}\right.$ and $\left.\sin ^{2} 2 \theta_{\mu \tau} \sim 1\right)$ as suggested by recent superkamiokande data concerning the deficit of atmospheric muon neutrino 11]. Taking the typical distance between the AGN and our galaxy as $L \sim 100 \mathrm{Mpc}$ (where $1 \mathrm{pc} \sim 3 \cdot 10^{16} \mathrm{~m}$ ), it follows that $P\left(\nu_{\mu} \rightarrow \nu_{\tau}\right) \sim 1 / 2$ for $2 \cdot 10^{6} \leq K / \mathrm{GeV} \leq 2 \cdot 10^{7}$, thus yielding $F\left(\nu_{\tau}+\bar{\nu}_{\tau}\right) \sim F\left(\nu_{\mu}+\bar{\nu}_{\mu}\right)$. Similarly, vacuum flavor oscillations between $\nu_{e}$ and $\nu_{\tau}$ may also take place for certain range of neutrino mixing parameters resulting in $P\left(\nu_{e} \rightarrow \nu_{\tau}\right) \sim \sin ^{2} 2 \theta_{e \tau}$.
} 


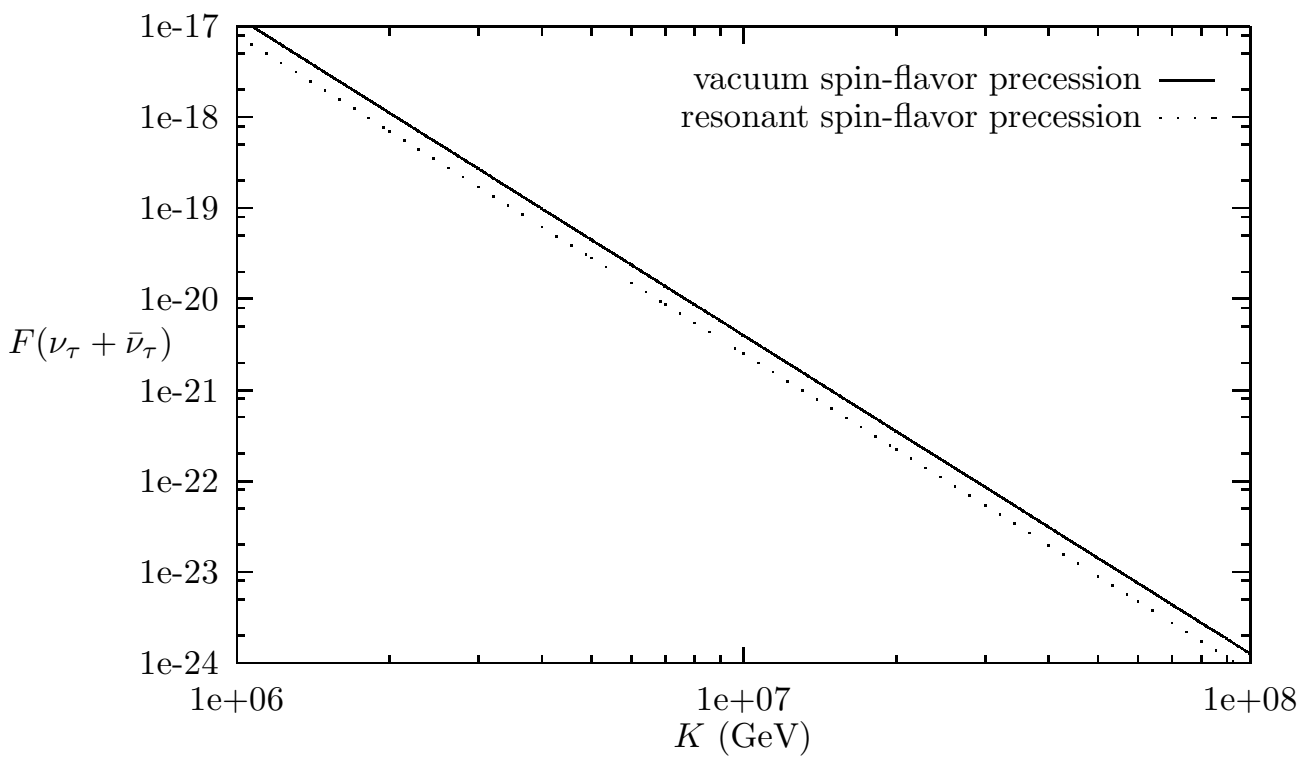

Figure 1: $F\left(\nu_{\tau}+\bar{\nu}_{\tau}\right)\left[\mathrm{cm}^{-2} \mathrm{~s}^{-1} \mathrm{sr}^{-1} \mathrm{GeV}^{-1}\right.$ ] Vs $K(\mathrm{GeV})$. The solid curve is obtained for $P \sim 0.8$ [using Eq. (4.12)], whereas the dotted curve is obtained using Eq. (4.14). In both cases, we use $\mu \sim 10^{-12} \mu_{B}$.

$B \rightarrow B_{T}$. Using then Eq. (4.8), the spin-flavor precession probability for constant $B$ and $\Delta V_{S F}$ is given by

$$
P\left(\nu_{e} \rightarrow \bar{\nu}_{\tau}\right)=\left[\frac{(2 \mu B)^{2}}{(2 \mu B)^{2}+\left(\Delta H_{S F}\right)^{2}}\right] \sin ^{2}\left[\sqrt{(2 \mu B)^{2}+\left(\Delta H_{S F}\right)^{2}} \frac{\Delta r}{2}\right] .
$$

Here $\Delta r$ is the width of the region where $B$ is appreciable. Let us study the various interesting situations that may arise from this expression for $P\left(\nu_{e} \rightarrow \bar{\nu}_{\tau}\right)$ under different physical conditions.

Let us consider first the case in which the contribution of the $\Delta V_{S F}$ term in Eq. (4.11) is small. If $\Delta m_{e \tau}^{2} / 2 K \ll$ $2 \mu B$, the above expression for $P\left(\nu_{e} \rightarrow \bar{\nu}_{\tau}\right)$ then reduces to

$$
P\left(\nu_{e} \rightarrow \bar{\nu}_{\tau}\right) \simeq \sin ^{2}(\mu B \Delta r),
$$

which is independent of $K$. Using the $B_{A G N}$ profile given by Eq. (4.2) and for $\mu \sim 10^{-12} \mu_{B}$ ( $\mu_{B}$ is Bohr magneton) [12], we obtain $P\left(\nu_{e} \rightarrow \bar{\nu}_{\tau}\right)>1 / 2$ for $\Delta m_{e \tau}^{2} \ll 10^{-2} \mathrm{eV}^{2}$. For this value of $\Delta m_{e \tau}^{2}\left(\sim 10^{-3} \mathrm{eV}^{2}\right)$, the resonant spinflavor precession due to matter induced gravity effects do not take place since the relevant level crossing condition is not satisfied. In contrast to the vacuum flavor oscillations mentioned earlier, the value of $P$ in this case can be different from $1 / 2$, thus raising the possibility of distinguishing between the two neutrino oscillation mechanisms. For $\Delta m_{e \tau}^{2} / 2 K \sim 2 \mu B$ or $\Delta m_{e \tau}^{2} / 2 K \gg 2 \mu B, P\left(\nu_{e} \rightarrow \bar{\nu}_{\tau}\right)$ is $K$ dependent and is such that $P\left(\nu_{e} \rightarrow \bar{\nu}_{\tau}\right) \leq 1 / 2$.

Taking into account now the effects of $\Delta V_{S F}$, the relevant level crossing condition $\left[\Delta H_{S F}=0\right.$, see Eq. (4.9)] can be satisfied for $\Delta m_{e \tau}^{2} \sim\left(10^{-8}-10^{-9}\right) \mathrm{eV}^{2}$. This relatively small value of $\Delta m_{e \tau}^{2}$ is also interesting in the context of supernova explosions 13 and the Sun[14]. Furthermore, this value of $\Delta m_{e \tau}^{2}$ is about 2 orders of magnitude larger than the one required for flavor level crossing [see Eq. (4.4)]. This is due to the fact that the nucleon contribution in $\Delta V_{S F}$ does not vanish (in contrast to that in $\Delta V_{F}$ ), as we emphasized earlier.

The adiabaticity condition at the level crossing in this case is [15]

$$
\kappa_{S F} \equiv \frac{2(2 \mu B)^{2}}{\left|\Delta \dot{V}_{S F}\right|} \geq 1
$$

Using the $B_{A G N}$ profile given by Eq. (4.2), this condition can be satisfied for $\mu \sim 10^{-12} \mu_{B}$, which is an order of magnitude smaller than the value that is needed in the context of the Sun 14. The general expression for $P\left(\nu_{e} \rightarrow \bar{\nu}_{\tau}\right)$, including the possible non-adiabatic effects $\left(\kappa_{S F}<1\right)$ is given by [16]

$$
P\left(\nu_{e} \rightarrow \bar{\nu}_{\tau}\right)=0.5-\left(0.5-P_{L Z}\right) \cos 2 \theta_{B},
$$


where $\tan 2 \theta_{B}=(-2 \mu B) / \Delta H_{S F}$ is being evaluated at the neutrino production site and $P_{L Z}=\exp \left(-\frac{\pi}{2} \kappa_{S F}\right)$. The expected $\nu_{\tau}$ flux spectrum due to (resonant) spin-flavor precession is calculated as

$$
F\left(\nu_{\tau}+\bar{\nu}_{\tau}\right)=P\left(\nu_{e} \rightarrow \bar{\nu}_{\tau}\right) F^{0}\left(\nu_{e}+\bar{\nu}_{e}\right)+\left[1-P\left(\nu_{e} \rightarrow \bar{\nu}_{\tau}\right)\right] F^{0}\left(\nu_{\tau}+\bar{\nu}_{\tau}\right) .
$$

A similar expression for $F\left(\nu_{\tau}+\bar{\nu}_{\tau}\right)$ due to flavor/spin-flavor oscillations in other channels can be straightforwardly obtained with appropriate changes. For $F^{0}\left(\nu_{e}+\bar{\nu}_{e}\right)$ we use the results from Ref. [9] for $K \geq 10^{6} \mathrm{GeV}$ as an example and, since $F^{0}\left(\nu_{\tau}+\bar{\nu}_{\tau}\right) / F^{0}\left(\nu_{e, \mu}+\bar{\nu}_{e, \mu}\right) \ll 1$, the explicit form of $F^{0}\left(\nu_{\tau}+\bar{\nu}_{\tau}\right)$ is not important here. For illustrative purposes, in Fig. 1, we display $F\left(\nu_{\tau}+\bar{\nu}_{\tau}\right)$ as a function of neutrino energy for various neutrino spin-flavor transition mechanisms, using Eq. (4.15). In the resonant spin-flavor precession case, since the adiabaticity condition [Eq. (4.13)] is satisfied, we have $P_{L Z} \sim 0$. Also, since the corresponding value of $\cos 2 \theta_{B}$ is quite small, $P\left(\nu_{e} \rightarrow \bar{\nu}_{\tau}\right) \sim 1 / 2$ [see Eq. (4.14)]. We have verified that a similar behavior of $P\left(\nu_{e} \rightarrow \bar{\nu}_{\tau}\right)$ results if the matter-induced gravity effects are omitted, but for a value of $\Delta m^{2}$ which, for the reason we mentioned earlier, is approximately 2 orders of magnitude smaller.

Another possibility, which we have not considered in detail, is that the transitions occur between active and sterile neutrinos. As in the case just discussed, the nucleon contribution in the Hamiltonian is important in this case also. In the presence of a magnetic field, the resonant spin-flavor transition may lead to energy dependent disappearance/appearance of the active neutrino fluxes even if one uses the stringent astrophysical upper bounds on the relevant transition magnetic moment 12 .

\section{Conclusions}

When neutrinos propagate in a medium composed of electrons and nucleons, their gravitational couplings are modified due to the weak interactions with the particles in the background. While the tree level gravitational interactions of the neutrinos have no effect on the oscillation phenomena, the matter-induced gravitational couplings depend on the background particle number densities and, in the presence of a gravitational potential, they lead to additional contributions to the neutrino indices of refraction which are not the same for the various neutrino flavors.

We have considered the effects that such matter-induced couplings may have on the phenomena associated with resonant neutrino oscillations, under the combined presence of a gravitational and a magnetic field. As an example of a setting where these effects are relevant, we have studied their influence on the determination of the flux of high energy neutrinos from the core of Active Galactic Nuclei. In that context we have pointed out that the resonant neutrino spin-flavor transitions may take place for $\Delta m^{2} \sim\left(10^{-8}-10^{-9}\right) \mathrm{eV}^{2}$ and $\mu \sim 10^{-12} \mu_{B}$, and due to the matter-induced gravity effects the estimated high energy tau neutrino flux is somewhat higher than it would be if those effects are not included. We have relied on the perturbative calculation of the matter-induced gravitational couplings carried out in Ref. [2]. According to the current interpretation of the observed photon flux from cores of AGN's, the neutrino production should take place for values of $x$ around $10-100$ as we have assumed in the text. In this case, the use of those perturbative results is justified and as a consequence the conditions for resonant transitions do not depend on the choice of any metric parameters unlike in Ref. [3].

We have set aside some possible incoherent effects which could be present for the high energy neutrinos that we are considering. The considerations of the present work indicate that more detailed calculations taking them into account are worth pursuing, and that they have useful applications in the context of neutrino emission in the vicinity of the core of AGN, with interesting implications for $\mathrm{km}^{2}$ surface area high energy neutrino telescopes.

Acknowledgments This work has been supported by a fellowship from the Japan Society for the Promotion of Science (HA) and by the U.S. National Science Foundation Grant PHY-9900766 (JFN).

\section{Appendix}

\section{A Equation for the amplitudes}

Here we derive Eq. (3.3). We begin by considering one neutrino, with both a left- and a right-handed component, propagating through the medium with momentum $k^{\mu}=(\omega, \vec{K})$, in the presence of a gravitational potential and a 
magnetic field $\vec{B}$. In a homogeneous medium, the dispersion relation and wavefunction of the propagating modes are determined from the linear part of the effective field equation, which in momentum space takes the form

$$
\left(\not k-m-\Sigma_{e f f}+\mu \vec{\Sigma} \cdot \vec{B}\right) \psi=0 .
$$

$\Sigma_{\text {eff }}$ is the self-energy of the neutrino, which in the situation that we are envisaging takes the form

$$
\Sigma_{e f f}=b_{L} \psi L+b_{R} \psi R,
$$

where

$$
b_{L}=b_{\text {mat }}+b_{G},
$$

with $b_{\text {mat }}$ and $b_{G}$ being given by the formulas quoted in Eqs. (3.7) and (3.8). $u^{\mu}$ stands for the velocity four-vector of the background medium. We adopt the frame in which the background is at rest, so that $u^{\mu}=(1, \overrightarrow{0})$.

The formula for $b_{R}$ depends on the nature of the right-handed component field. If it is the conjugate field of a standard left-handed neutrino, then $b_{R}=-b_{L}$. However, for a weak singlet field, which we assume to be the case in what follows, $b_{R}=0$. In Eq. A.2 we are neglecting terms of order $g^{2} / m_{W}^{4}$, as well as the magnetic-field dependent term that arises from the effective electromagnetic interactions of the neutrino in matter [17]. The latter contribution is not significant for the values of the magnetic field that we are considering here. In addition, we have dropped the purely gravitational term denoted by $b_{g}$ in Ref. [2], which has a universal value for all the neutrinos (including the sterile ones) and therefore is not relevant for neutrino oscillations.

Writing

$$
\psi=\left(\begin{array}{l}
\xi \\
\eta
\end{array}\right)
$$

in the Weyl representation of the gamma matrices, Eq. (A.1) becomes

$$
\begin{aligned}
\left(\omega-b_{L}+\vec{\sigma} \cdot \vec{K}\right) \eta-m \xi+\mu(\vec{\sigma} \cdot \vec{B}) \xi & =0, \\
(\omega-\vec{\sigma} \cdot \vec{K}) \xi-m \eta+\mu(\vec{\sigma} \cdot \vec{B}) \eta & =0,
\end{aligned}
$$

where $\vec{\sigma}$ are the Pauli matrices. Consider first the case in which $\vec{B}=0$. In this case, the equations have non-trivial solutions only if $\xi$ and $\eta$ are proportional to the same spinor $\phi_{\lambda}$ with definite helicity. The positive energy solutions are then $\omega_{-} \simeq K+b_{L}+\frac{m^{2}}{2 K}$ and $\omega_{+} \simeq K+\frac{m^{2}}{2 K}$ for $\lambda=-1$ and $\lambda=+1$, respectively, with the corresponding Dirac wavefunctions

$$
\begin{aligned}
& \psi_{-}=\psi_{L}+y\left(\begin{array}{c}
\phi_{-} \\
0
\end{array}\right), \\
& \psi_{+}=\psi_{R}+y^{\prime}\left(\begin{array}{c}
0 \\
\phi_{+}
\end{array}\right),
\end{aligned}
$$

where $y, y^{\prime}$ are of order $m / K$, and

$$
\psi_{L}=\left(\begin{array}{c}
0 \\
\phi_{-}
\end{array}\right), \quad \psi_{R}=\left(\begin{array}{c}
\phi_{+} \\
0
\end{array}\right)
$$

When $\vec{B} \neq 0$, it is convenient to decompose $\vec{B}$ according to

$$
\vec{B}=B_{\|} \hat{K}+\vec{B}_{T} .
$$

Then it is easy to see that, if $\vec{B}_{T} \neq 0$, the two helicity spinors get mixed in Eq. A.5), and therefore we put

$$
\begin{aligned}
& \eta=\alpha \phi_{-}+\epsilon \phi_{+}, \\
& \xi=\beta \phi_{+}+\epsilon^{\prime} \phi_{-} .
\end{aligned}
$$

Substituting Eq. (A.9) into Eq. (A.5) it follows that $\epsilon$ and $\epsilon^{\prime}$ are of order $m / K$ and $\mu B_{\|} / K$, while the equations that are obtained for $\alpha$ and $\beta$ can be recast in the form

$$
H\left(\begin{array}{c}
\alpha \\
\beta
\end{array}\right)=\omega\left(\begin{array}{c}
\alpha \\
\beta
\end{array}\right),
$$


where

$$
H=\left(\begin{array}{cc}
K+b_{L}+\frac{\left(m+\mu B_{\|}\right)^{2}}{2 K} & -\mu B_{T} \\
-\mu B_{T} & K+\frac{\left(m-\mu B_{\|}\right)^{2}}{2 K}
\end{array}\right) .
$$

For a given solution of Eq. (A.10), the Dirac wavefunction of the corresponding mode is obtained from Eqs. (A.4) and (A.9). Neglecting terms proportional to $\epsilon$ and $\epsilon^{\prime}$, it is then given by

$$
\psi=\alpha \psi_{L}+\beta \psi_{R},
$$

where $\psi_{L, R}$ are defined in Eq. (A.7). For an inhomogeneous medium, $H$ is taken as the Hamiltonian for the amplitudes $\alpha, \beta$.

The generalization to two or more families is straightforward. The same procedure yields an equation for the amplitudes that is identical to Eq. (A.10), but where each element of $H$ is considered to be a matrix in the neutrino flavor space. Similarly, the amplitudes $\alpha$ and $\beta$ are spinors in that flavor space, and they determine the spin and flavor content of the corresponding propagating mode according to Eq. (A.12). These are the results quoted in Eqs. (3.2) and (3.3).

In the second case that we consider, specified by the interaction Lagrangian terms given in Eqs. (3.12) and (3.13), the field equations, including the matter effects, are

$$
\begin{aligned}
\left(\not k-b_{L}^{\left(\nu_{e}\right)} \not\right) \nu_{e L}-m_{\nu_{e}} N_{e R}+\mu(\vec{\Sigma} \cdot \vec{B}) \nu_{\tau R}^{c} & =0, \\
\not k N_{e R}-m_{\nu_{e}} \nu_{e L} & =0, \\
\left(\not k-b_{R}^{\left(\bar{\nu}_{\tau}\right)} \not\right) \nu_{\tau R}^{c}-m_{\nu_{\tau}} N_{\tau L}^{c}+\mu(\vec{\Sigma} \cdot \vec{B}) \nu_{e L} & =0, \\
\not k N_{\tau L}^{c}-m_{\nu_{\tau}} \nu_{\tau R}^{c} & =0 .
\end{aligned}
$$

where 18

$$
b_{R}^{\left(\bar{\nu}_{\tau}\right)}=-b_{\text {mat }}^{\left(\nu_{\tau}\right)}-b_{G}^{\left(\nu_{\tau}\right)} .
$$

Restricting ourselves for the moment to the situation in which $B_{\|}=0$, the positive energy solutions of these equations are found by putting, in the Weyl representation,

$$
\begin{aligned}
\nu_{e L} & =\alpha_{\nu_{e}}\left(\begin{array}{c}
0 \\
\phi_{-}
\end{array}\right), \\
N_{e R} & =\epsilon\left(\begin{array}{c}
\phi_{-} \\
0
\end{array}\right), \\
\nu_{\tau R}^{c} & =\beta_{\bar{\nu}_{\tau}}\left(\begin{array}{c}
\phi_{+} \\
0
\end{array}\right), \\
N_{\tau L}^{c} & =\epsilon^{\prime}\left(\begin{array}{c}
0 \\
\phi_{+}
\end{array}\right) .
\end{aligned}
$$

Substituting these forms into Eq. A.13), this procedure yields

$$
\begin{aligned}
\epsilon & \simeq \frac{m_{\nu_{e}}}{2 K} \alpha_{\nu_{e}}, \\
\epsilon^{\prime} & \simeq \frac{m_{\nu_{\tau}}}{2 K} \beta_{\bar{\nu}_{\tau}},
\end{aligned}
$$

while the equations for $\alpha_{\nu_{e}}$ and $\beta_{\bar{\nu}_{\tau}}$ can be written in the form

$$
H\left(\begin{array}{c}
\alpha_{\nu_{e L}} \\
\beta_{\bar{\nu}_{\tau R}}
\end{array}\right)=\omega\left(\begin{array}{c}
\alpha_{\nu_{e L}} \\
\beta_{\bar{\nu}_{\tau R}}
\end{array}\right)
$$

with

$$
H=\left(\begin{array}{cc}
K+b_{\mathrm{mat}}^{\left(\nu_{e}\right)}+b_{G}^{\left(\nu_{e}\right)}+\frac{m_{\nu_{e}}^{2}}{2 K} & -\mu B_{T} \\
-\mu B_{T} & K-b_{\text {mat }}^{\left(\nu_{\tau}\right)}-b_{G}^{\left(\nu_{\tau}\right)}+\frac{m_{\nu_{\tau}}^{2}}{2 K}
\end{array}\right)
$$

When $B_{\|} \neq 0$, the components of the wave function shown in Eq. A.15) acquire an admixture of the opposite helicity spinor. Allowing this and carrying through the same steps as before, the resulting equation for $\alpha_{\nu_{e}}$ and $\beta_{\bar{\nu}_{\tau}}$ can be written once again as in Eq. (A.17), but with $H$ given by the formula quoted in Eq. (3.15). 


\section{References}

[1] For a recent review, George G. Raffelt, hep-ph/9902271. See also, R. J. Protheroe, Nucl. Phys. B (Proc. Suppl.) 77, 465 (1999).

[2] José F. Nieves and Palash B. Pal, Phys. Rev. D58, 096005 (1998).

[3] Dardo Píriz, Mou Roy and José Wudka, Phys. Rev. D54, 1587 (1996).

[4] In this respect, our results differs in a crucial way from that of Ref. [3]. There, the tree-level gravitational couplings were found to yield different contributions to the left and to the right-handed neutrino dispersion relations, while the matter effects on the gravitational couplings were completely neglected. As explained here, we disagree with that treatment.

[5] As usual, for any left-handed field $\nu_{L}, \nu_{R}^{c}$ stands for the Lorentz-invariant complex conjugate, i.e., for any flavor $f, \nu_{f R}^{c}=i \gamma_{2} \nu_{f L}^{*}$, in the standard representation of the gamma matrices.

[6] The same result is obtained if, instead of Eq. (3.13), the mass terms are of the form $m_{\nu_{e}} \bar{\nu}_{e R}^{c} \nu_{e L}$ and similarly for $\nu_{x}$, so that $\nu_{e}$ amd $\nu_{x}$ are Majorana particles. Without the matter and gravitational effects this is the case considered in J. Schechter and J. W. F. Valle, Phys. Rev. D24, 1883 (1981). The matter effects were considered in the papers cited in Ref. [15].

[7] A more detailed numerical study supports this estimate; H. Athar, R. A. Vázquez and E. Zas, in preparation.

[8] J. G. Learned and S. Pakvasa, Astropart. Phys. 3, 267 (1995); F. Halzen and D. Saltzberg, Phys. Rev. Lett. 81, 4305 (1998). See also, S. Bottai and F. Becattini, in 26th International Cosmic Ray Conference, Salt Lake City, 17-25 August (to appear in its proceedings); S. Iyer, M. H. Reno and I. Sarcevic, hepph/9909393. For information on current status of various high energy neutrino telescopes, see, for instance, L. Moscoso in Sixth International Workshop on Topics in Astroparticle and Underground Physics (TAUP 99), Paris (France), September 1999 (to appear in its proceedings), scanned transperacies are available at URL http://taup99.in2p3.fr/TAUP99/Thursday/thursday.htm.

[9] A. P. Szabo and R. J. Protheroe, Astropart. Phys. 2, 375 (1995).

[10] S. P. Mikheyev and A. Yu. Smirnov, Sov. J. Nucl. Phys. 42, 913 (1985).

[11] Y. Fukuda et al., Phys. Rev. Lett. 81, 1562 (1998); Phys. Lett. B433, 9 (1998); 436, 33 (1998).

[12] G. G. Raffelt, Phys. Rev. Lett. 64, 2856 (1990).

[13] H. Athar, J. T. Peltoniemi and A. Yu. Smirnov, Phys. Rev. D51, 6647 (1995).

[14] For a recent update, see, for instance, M. M. Guzzo and H. Nunokawa, Astropart. Phys. 12, 87 (1999).

[15] C.-S. Lim and W. J. Marciano, Phys. Rev. D37, 1368 (1988); E. Kh. Akhmedov, Sov. J. Nucl. Phys. 48, 382 (1988); Phys. Lett. B213, 64 (1988).

[16] See, for instance, George G. Raffelt in Ref. 11] and references cited therein.

[17] J. F. Nieves and P. B. Pal, Phys. Rev. D40, 1693 (1989); J. C. DÓlivo, J. F. Nieves, P. B. Pal, ibid.40, 3679 (1989); J. C. DÓlivo, J. F. Nieves, P. B. Pal, Phys. Rev. Lett. 64, 1088 (1990). See also, V. B. Semikoz and J. W. F. Valle, Nucl. Phys. B425, 651 (1994); S. Esposito and G. Capone, Z. Phys. C70, 55 (1996); H. Nunokawa, V. B. Semikoz, A. Yu. Smirnov amd J. W. F. Valle, Nucl. Phys. B501, 17 (1997).

[18] In general the coefficients $b^{\left(\nu_{\alpha}\right)}$ are functions of $\omega$ and $\vec{K}$. In those cases, the relation is $b_{R}^{\left(\bar{\nu}_{\alpha}\right)}(\omega, \vec{K})=$ $-b_{L}^{\left(\nu_{\alpha}\right)}(-\omega,-\vec{K})$. 